\title{
Alter
}

Revue de phénoménologie

$27 \mid 2019$

Patočka

\section{Homo respondens}

\section{Bernhard Waldenfels}

Traducteur : Audran Aulanier

\section{OpenEdition}

\section{Journals}

Édition électronique

URL : https://journals.openedition.org/alter/1932

DOI : $10.4000 /$ alter. 1932

ISSN : 2558-7927

Éditeur :

Association ALTER, Archives Husserl (CNRS-UMR 8547)

Édition imprimée

Date de publication : 1 novembre 2019

Pagination : 249-261

ISBN : 978-2-9550449-5-7

ISSN : 1249-8947

\section{Référence électronique}

Bernhard Waldenfels, « Homo respondens », Alter [En ligne], 27 | 2019, mis en ligne le 22 décembre 2020, consulté le 14 juin 2021. URL : http://journals.openedition.org/alter/1932 ; DOI : https://doi.org/ 10.4000/alter.1932

Ce document a été généré automatiquement le 14 juin 2021.

Revue Alter 


\title{
Homo respondens ${ }^{1}$
}

\author{
Bernhard Waldenfels
}

Traduction : Audran Aulanier

1 L'homme est un être qui se remet lui même en question. La question « qui suis-je? » ne se laisse pas mettre de côté, pas plus que le «ici» et le «maintenant» de ce propos. Une anthropologie qui tente d'éradiquer tout reste d'égologie se fige inévitablement dans une idéologie qui nous laisse dans l'obscurité quant à l'origine des idées. Mais puisque chaque discours s'adresse implicitement ou explicitement à quelqu'un, la question « qui suis-je ? » se dédouble en un « qui es-tu? ». Tout cela n'a rien à voir avec un amour-propre narcissique de l'homme, mais découle du fait que chaque question, y compris la question anthropologique, a un lieu d'où elle surgit. Les questions ne tombent pas du ciel.

\section{L'homme multiforme}

2 Le questionnement sur soi implique que l'homme apparaît dans différents rôles et avec un nombre d'épithètes à n'en pas finir. Depuis Linné, en tant qu'homo sapiens, il a sa place dans la structure ancestrale de la nature : un être sensé qui surpasse physiquement l'homo erectus et qui émerge du clair-obscur des singes anthropoïdes et des hommes singes. Comme homo faber, il se distingue par son habileté et l'utilisation du bronze ou de la pierre, comme homo laborans il se livre à un travail pénible, comme homo ludens il met en œuvre ses forces ludiques, comme homo pictor il se transforme luimême et son monde en images, en commençant par les premiers dessins rupestres. ${ }^{2} S^{\prime} y$ ajoutent des constructions telles que l'homo oeconomicus et des produits des alchimistes tels que l'homoncule. Et maintenant un autre, l'homo respondens? L'homme en tant qu'être répondant nous rappelle certainement la vieille définition aristotélicienne de l'homme en tant qu'être vivant, doté d'un Logos et vivant avec d'autres hommes dans une Polis. Mais avec la réponse, nous mettons notre propre accent. Si chaque mot de la langue est un "mot semi-étranger $»^{3}$, ceci est particulièrement vrai pour la réponse. La voix du répondant est pro-voquée, elle vient d'ailleurs; on répond à quelque chose ou à quelqu'un. Le "à quoi" (Worauf) de la réponse ne doit pas être confondu avec le "ce à 
propos de quoi" (Worüber) d'une déclaration, ou avec la raison pour laquelle (Wozu) je prends une décision. La réponse ne vient pas de moi. Celui qui apparaît au grand jour dans la réponse se met en travers des définitions courantes. Il n'est pas un simple « être imparfait » qui a un manque à compenser, il ne se distingue pas non plus comme « roi de la création ", pas plus qu'il n'habite " au milieu du monde ». Il s'avère plutôt être un " être intermédiaire » qui construit des ponts et qui, en tant qu' «animal qui n'est pas encore fixé de manière stable $»^{4}$, provoque des troubles dans le monde par sa recherche d'un lieu.

\section{L'énigme du Sphinx}

3 La recherche d'un lieu par l'homme se reflète dans de nombreux mythes des origines. Parmi eux se trouve une ancienne énigme de l'antiquité grecque, qui forme l'arrièreplan mythologique de la tragédie d'œEdipe. Cette histoire ne commence pas comme une intrigue dramatique, elle naît d'un pathos. La ville de Thèbes, qui est en proie à la peste, a déjà souffert des horreurs du Sphinx. Ce monstre ailé cherchait ses victimes en leur posant une énigme : "Quel est l'être qui marche le matin sur quatre pattes, à midi sur deux pattes et le soir

sur trois pattes?»; quiconque ne répondait pas était dévoré par le monstre. CEdipe, qui a été abandonné par son père après sa naissance et que son nom, " pieds enflés ", trahit comme étant quelqu'un dont le propre pas est entravé, sauve la ville. Il résout l'énigme, brisant le sort en prononçant le nom «homme »: «Tu parles de l'homme - öv $\theta \rho \omega \pi$ ov $\kappa \alpha \tau \varepsilon ́ \lambda \varepsilon \xi \alpha \varsigma$ (ánthropon katélexas)... » La formulation de la solution ternit les qualités du Logos et de la Polis en dépeignant l'homme comme un mortel, un être vieillissant dont la vie commence par l'impuissance de l'enfant et se termine dans la décrépitude de la vieillesse. «Allbewandert. Unbewandert zu nichts kommt er. Der Toten künftigen Ort nur zu fliehen weiß er nicht. $»^{5}$. C'est le passage traduit par Hölderlin du chant du chœur d'Antigone (v. 360-362), dans lequel le «monstrueux » de l'homme est évoqué ${ }^{6}$. Mais la scène de l'énigme révèle encore davantage. Nous passons à côté de sa leçon lorsque nous érigeons Edipe en héros explorateur ou, inversement, quand nous rapprochons le mystère du Sphinx d'une question de quiz. Comme le révèle le prologue du Roi đdipe (v. 38), Æedipe n'a pas trouvé la bonne solution sans «l'aide d'un dieu -

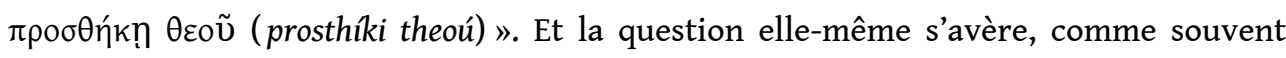
dans les contes, être une question de vie ou de mort, dont la réponse exige plus qu'une simple perspicacité. La suite de la tragédie montre les abîmes du parricide et de l'inceste dans lesquels dérive $\mathbb{E}$ dipe avec son infatigable soif de recherche. On sait combien l'idée freudienne de descente ad inferos doit à la lecture intensive de la tragédie grecque ${ }^{7}$.

4 Nous avons tendance à désamorcer ces vieux textes. Ne viennent-ils pas des temps prémodernes marqués par un manque d'autodétermination, et dans lesquels les dieux et les créatures mythiques déterminaient encore notre destin ? La personne émancipée ne se distingue-t-elle pas en posant des questions au lieu de répondre aux questions des autres? Déjà à l'Antiquité, Prométhée semble apparaître plutôt comme patriarche de l'humanité. Dans la deuxième préface de la Critique de la raison pure (B XII), Kant fait l'éloge des naturalistes modernes comme Galilée et Torricelli : «Ils comprirent que la raison [... doit] forcer la nature à répondre à ses questions, au lieu de se laisser conduire par elle comme à la lisière $[. ..] »^{8}$. La liberté, qui distingue l'homme en tant qu'être de 
raison, signifie commencer par soi-même. Le soi est capitalisé sous la forme d'une autonomie morale et politique et maintenant aussi sous la forme systémique d'une autopoiesis. Celui qui prône un Heteron semble revenir à un état de dépendance immature et se déplacer à quatre pattes, pour ainsi dire, au lieu de progresser debout. Mais il n'en est pas moins certain que la modernisation forcée révèle ses côtés obscurs jusqu'au point où l'homme est dépassé par ses propres succès. Les Grecs, qui pensèrent par avance à l'utilisation d'outils automatiques avec les statues de Dédale ${ }^{9}$, ont utilisé la chute d'Icare comme un avertissement précoce. Face à un "projet de la Modernité " chancelant, la tentation est grande d'emprunter les routes de la contre-modernité : même l'histoire a ses automobilistes qui roulent à contre-sens. Mais de simples demitours tels que la restauration plutôt que la révolution, la conservation plutôt que l'innovation n'ont jamais été couronnés de succès. La responsivité qui nous préoccupe ne signifie pas un revirement à l'opposé, mais un rééquilibrage qui implique un «devenir-étranger (Verfremdung) de la modernité $»^{10}$. L'homme répondant n'est ni maitre des choses ni leur jouet. Pour le montrer, les principales caractéristiques d'une phénoménologie responsive sont esquissées ci-dessous. Ceci est fait sans intention de résoudre l'énigme du sphinx, mais plutôt de redonner à l'expérience son caractère énigmatique.

\section{Réponses techniquement standardisées, normales et créatives}

5 Mais de quel genre de réponse s'agit-il chez l'Homo respondens? Le répondre ne jouit généralement pas d'une grande considération. Il semble n'exister que pour combler les lacunes des connaissances propres et étrangères, ce qui présuppose déjà un cadre de connaissances. La bonne réponse serait de le découvrir, il ne reste pas grand-chose à inventer. Les choix multiples ne laissent pas beaucoup de choix. Si un répertoire de réponses (Antwortrepertoire) est disponible, la réponse peut être récupérée ; avec un formatage suffisant, un appareil répondant suffit. Un programme informatique peut faire des diagnostics thérapeutiques et donner des conseils. Toutefois, la condition préalable est que les plaintes des patients ne dépassent pas le champ d'application de la réglementation et respectent les formats et formulaires standardisés ${ }^{11}$. Outre les réponses techniquement préparées, il y a des réponses normales qui font partie de notre vie quotidienne ; elles sont utiles et indispensables à la conversation, mais elles vivent des réserves de notre praxis quotidienne, qui sont seulement mises en œuvre et testées dans le monde du travail. Il en va de même pour le quotidien des institutions, c'est-àdire aussi pour la recherche quotidienne dans les sciences normales. Enfin, les réponses sont réduites à de simples réactions lorsqu'elles sont définies comme un effet d'un stimulus et conditionnées en conséquence, conformément au schéma behavioriste de stimulus / réponse. Rien ne change fondamentalement si le modèle linéaire est remplacé par un circuit fermé et si les réponses données sont stimulées rétroactivement, comme avec le thermostat. Cela ne devient sérieux que lorsque le processus normal est perturbé et lorsque les habitudes et les programmes d'intervention échouent ${ }^{12}$. Dans de tels cas, il faut des réponses créatives qui mettent en jeu quelque chose de nouveau. Mais la question se pose alors de savoir dans quelle mesure une réponse peut être créative en tant que réponse et une création responsive en tant que création. 


\section{La responsivité en tant que caractéristique fondamentale du comportement}

$6 \quad$ Lorsque nous parlons de responsivité dans ce qui suit, il ne s'agit pas de comportements particuliers tels que donner des informations ou répondre à une question lors d'un examen, mais d'une caractéristique fondamentale qui façonne l'ensemble de notre comportement corporel et fait ainsi usage d'une «ingéniosité du corps » ${ }^{13}$. Regarder, écouter, fantasmer, sourire ou sentir sont aussi affectés que parler, faire ou produire. Répondre signifie que nous entrons dans l'étranger, qui ne se laisse pas surmonter par les moyens existants du propre et du commun. J'ai moi-même emprunté le terme de responsivité au langage de la médecine et plus précisément à la façon de parler de l'école de Virchow. Le neurologiste juif allemand Kurt Goldstein comprend la responsivité comme la capacité de l'organisme ou d'un individu à répondre adéquatement aux exigences d'un milieu, et il décrit comme une irresponsivité la déficience pathologique de cette capacité. Goldstein, qui a dirigé un centre de réhabilitation à Francfort dans l'entre-deux-guerres jusqu'à son émigration forcée, a passé des années à étudier, avec ses collègues, la manière dont une lésion cérébrale causée par un fragment de grenade dans la zone optique a altéré la responsivité du comportement global chez le patient Schneider, tout en essayant de mettre en place une thérapie responsive ${ }^{14}$. On peut en trouver des traces dans les histoires médicales d'oliver Sacks. L'homme qui confond sa femme avec un chapeau mène aux bords pathologiques d'un être humain incertain.

7 La capacité de réponse, qui peut être distinguée de la responsabilité de l'action, a depuis longtemps trouvé son chemin dans les pratiques sociales. Mais les théories habituelles de l'action et du langage se contentent généralement d'examiner les objectifs, les règlements et les circonstances pragmatiques sans poser la question de savoir ce à quoi quelqu'un répond lorsqu'il dit ou fait ceci ou cela. Mais c'est seulement avec cette question que nous entrons dans ce que Kant appelle le «fertile bathos de l'expérience $»^{15}$. Quiconque se hâte d'aller au niveau du jugement et de la décision prétend que la vie se déroule dans une salle d'audience imaginaire. La créativité de l'homme répondant est négligée dans une telle orthologie et orthopraxie ${ }^{16}$.

\section{Pathos...}

Cela nous amène au cœur de nos considérations. La responsivité, qui détermine le cours de notre expérience, se présente comme un double événement de pathos et de réponse. Par l'expression grecque Pathos ou l'expression allemande Widerfahrnis je comprends le fait originaire que quelque chose nous frappe, vient à nous, attire l'attention ou vient à l'esprit ${ }^{17}$, que quelque chose réussisse à nous atteindre et aussi à nous blesser, comme le touché $^{18}$ du combat d'escrime. Le surprenant et l'inhabituel peuvent résulter de changements minimes qui ont un effet profond. Ils se manifestent de manière significative sous la forme d'un flash soudain, d'une explosion ou d'un choc. Ils peuvent apparaître à proximité ou à distance, comme l'illumination d'une nouvelle étoile ou comme le Big Bang, daté avec bien des difficultés. Le changement peut être basé sur des paroles et des pensées comme Nietzsche l'avait à l'esprit: "Les paroles les plus silencieuses sont celles qui apportent la tempête. Les pensées portées sur des pattes de 
colombe mènent le monde. $\aleph^{19}$. Notre histoire personnelle est scandée par des événements uniques tels que la naissance et la maturité, le choix du partenaire et sa perte, l'entrée dans la carrière, le changement de profession et la perte d'emploi, la maladie et la mort. L'histoire publique serait un tumulte infini de faits et de conditions sans bouleversements qui font date comme la Renaissance, la Réforme ou la Révolution, sans événements de rupture comme le déclenchement d'une guerre, un krach boursier ou une catastrophe naturelle, sans innovations techniques comme l'introduction de l'Internet ou de nouveaux départs artistiques comme l'invention de la perspective centrale, le déchaînement impressionniste de la couleur ou la transition vers la musique atonale. Certains événements ont des données de lieu et de temps fixes : New York le 11 septembre 2001 ou Fukushima le 11 mars 2011, tandis que d'autres changements commencent à émerger avant qu'ils ne remontent à la surface. Comme pour les maladies aiguës et chroniques, il y a des changements de scène aigus et chroniques. Dans tous les cas, ce sont des formes fortes d'expérience dans lesquelles non seulement quelque chose change dans le monde et dans nos vies, mais par lesquelles le monde et la vie dans son ensemble sont restructurés ou perturbés.

L'effraction ou l'infiltration du nouveau nous confronte à des événements qui s'écartent de l'habituel et, dans le cas extrême, nous bouleversent. Mais derrière la variété d'événements qui sont indiqués ici se cache une structure d'événement d'un type particulier. Le Pathos ou le Widerfahrnis ne doivent pas être confondus avec des événements observables, ils ne peuvent être vus que du point de vue du participant. Ce qui m'arrive à moi, à toi, à nous ou à d'autres se manifeste par un effet corporel en nous affectant, littéralement: il nous fait mal ou nous excite, et il nous interpelle en nous exhortant. De forts effets produisent un surplus affectif à travers l'étonnement, l'épouvante ou la stupeur. Platon fait commencer la philosophie avec un étonnement qui nous donne le vertige et qui est proche de l'horreur. Avec une telle initiation, nous franchissons un seuil d'étrangeté. L'étonnement ne commence pas dans le propre comme le doute méthodique de Descartes, et il ne s'apprend pas non plus. Lichtenberg, qui s'est surpris lui-même à plusieurs reprises en écrivant son "Sudelbücher ", a des idées similaires en tête lorsqu'il recommande de dire "ça pense », comme on dit « ça clignote »; et Nietzsche est d'accord avec cela lorsqu'il déclare qu'« une pensée vient quand "elle" veut, et non pas quand "je" veux » ${ }^{20}$. Ce qui attire notre attention ou nous vient à l'esprit, nous ne l'avons jamais complètement entre nos mains. Grammaticalement, des verbes tels que " widerfahren », " auffallen » ou " einfallen » ${ }^{21}$ ne peuvent pas être utilisés activement ; ils ne doivent pas être compris comme des actes que nous considérons comme notre propre réalisation. Ils ressemblent en cela aux processus de réveil et d'endormissement qui déploient leur effet métaphorique en « sommeil dogmatique » ou en "réveil religieux ». Les désirs d'action d'un sujet sont ainsi atténués, mais ce n'est pas une raison pour plonger tête baissée dans un courant vital. Quelqu'un est impliqué à chaque fois que quelque chose nous arrive ou nous heurte (Widerfahrnis), non pas dans le nominatif de l'auteur mais dans le datif ou l'accusatif d'un patient, qu'il faut comprendre dans un sens plus large : « quelque chose me frappe ", "quelque chose m'a atteint ». Un Widerfahrnis qui se produirait sans que quelqu'un ne soit frappé serait comme une douleur que personne ne ressent. Nous sommes impliqués, mais pas en tant que sujets autocratiques (selbstherrliche). 


\section{... et réponse}

10 Mais ce n'est qu'un côté de la médaille. Le Widerfahrnis resterait sans effet et irréel s'il n'était pas exprimé ou parlé. Il parvient à l'expression, non pas comme quelque chose dont nous parlons, mais comme quelque chose auquel nous répondons. Dans cet interstice, le caractère répondant de la réponse (Antwortcharakter der Response) se déploie. Répondre signifie parler à partir de l'étranger. Cela me transforme d'un patient en un répondant qui répond à ce qui lui arrive ou le heurte (widerfährt). Le soi propre est un soi divisé. Parler d'un Homo respondens ne signifie pas seulement que l'homme est un être qui peut répondre et qui est prêt à répondre, mais qu'il devient homme en répondant, tout comme Edipe devient le sauveur de la ville en résolvant l'énigme. La réponse précède sa propre habilitation.

11 La réponse n'est pas à notre discrétion; elle découle d'une inévitabilité que nous avons déjà rencontrée dans l'énigme du Sphinx. Si quelque chose nous interpelle, nous ne pouvons pas ne pas répondre, tout comme Paul Watzlawick dit que nous ne pouvons pas ne pas communiquer. Nous sommes dans une sorte de piège responsif. Ne pas donner de réponse serait aussi une réponse, comme le dit l'adage. La contrainte de répondre ne signifie pas, bien sûr, que nous étions prêts à répondre. Chaque appel laisse une marge de manœuvre. Ce n'est pas à nous de décider si nous répondons, mais comment nous répondons. Si notre expérience s'exposait seulement à la foudre de l'instant, nous n'aurions aucune expérience. Dans nos réponses, le à quoi (Worauf) du répondre se transforme en quoi (Was) d'une réponse. Ce qui nous arrive ou nous heurte (Was uns widerfährt) prend une forme répétable, par exemple sous la forme d'un contraste de couleurs ou d'une séquence de sons; du sens est gagné, cela construit des règles et une disposition à répondre appropriée aux exigences changeantes. Ce processus de transformation peut s'enliser ou échouer.

12 La phrase de Goethe « un Dieu m'a donné de pouvoir dire combien je souffre »"2 signifie plus que la capacité de parler de sa propre souffrance ; il s'agit du fait que la souffrance franchit le seuil du silence, qu'elle vient à la parole, que les mots se trouvent aussi pour

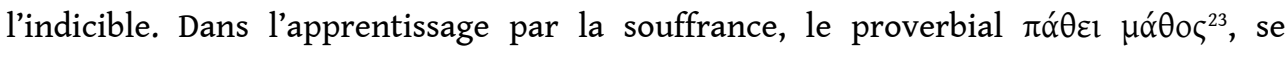
déroule ce qui s'appelle l'élaboration (Verarbeitung) en psychanalyse. La gestion de la souffrance fait partie du travail quotidien de la clinique. Le médecin transforme la souffrance du patient en une maladie qui présente des symptômes typiques, qui suit un cours typique, qui peut être traitée et guérie ou du moins soulagée. La thérapie est responsive si non seulement elle rétablit un état normal comme dans la réparation d'une machine, mais aussi si elle relance la capacité de répondre dans des conditions différentes et si elle brise les blocages de réponse. Cela s'applique d'une manière particulière au traitement thérapeutique des personnes traumatisées qui sont pratiquement réduites au silence et dont la blessure s'échappe par le langage corporel des symptômes. Mais il en va de même pour des réponses collectives à grande échelle. La politique lancée à la suite de la catastrophe nucléaire de Fukushima, par exemple, est une réponse plutôt qu'un simple nouveau départ. Si cette réponse ne fonctionne pas, alors c'est aussi une réponse, mais pas de celles qui déclenchent un processus d'apprentissage. Le travail de mémoire imposé aux allemands par la politique du Troisième Reich et par les atrocités de l'Holocauste a aussi un caractère responsif. Sans la volonté publique de répondre, les monuments commémoratifs et les 
commémorations n'auraient rien d'autre à offrir que des répétitions vides d'où s'échapperait l'inrenouvelable.

Pathos et réponse sont comme deux maillons d'une chaîne qui ne se ferme pas. L'un ne peut être dérivé de l'autre. Un pathos sans réponse resterait à jamais silencieux et serait tôt ou tard oublié. Une réponse sans pathos serait une phrase vide de sens ou un simple exercice de devoir. La créativité est une réponse inventive; elle invente ce qu'elle donne comme réponse, mais pas ce à quoi elle doit répondre. Cela diffère à la fois d'un fondamentalisme qui prétend fournir des réponses toutes faites, et d'un constructivisme qui tronque les contradictions en de simples données de base.

\section{Diastase}

Une expérience qui se déplace entre le pathos et la réponse présente une structure temporelle particulière. Les événements surprenants ont en eux-mêmes le fait qu'ils arrivent trop tôt par rapport à nos attentes, sinon ils ne seraient pas surprenants. Seuls les événements normaux qui répondent à nos attentes et à nos plans arrivent plus ou moins à temps, y compris les retards éventuels comme dans le cas du tourisme. De tels retards peuvent être corrigés, mais cela ne s'applique pas aux surprises, à moins que l'on ne s'efface en s'enfuyant dans l'apathie. Donc, si ce qui nous arrive ou nous heurte vient trop tôt, notre réponse arrivera trop tard en regard de l'exigence qui nous réclame. Je désigne la double non-simultanéité de l'avance originaire du pathique et du retard originaire de la réponse comme Zeitverschiebung ${ }^{24}$ ou avec une ancienne expression grecque comme diastase. L'expérience se désintègre littéralement, elle se distend. Ce décalage temporel particulier ne peut pas être compris comme une suite de points sur une ligne temporelle ni comme une causalité linéaire, comme si l'expérience venait d'abord et que la réponse suivait ensuite. Au contraire, l'expérience se précède elle-même ; en tant que répondants, nous sommes impliqués dès le début, mais pas en tant qu'instigateurs.

15 Le décalage temporel apparaît sous de nombreuses formes. Même ma naissance appartient à un passé qui n’a jamais été vécu comme un présent, et pourtant c'est moi qui me retrouve né. Cela se répète partout où des choses nouvelles émergent dans l'histoire, pour lesquelles on ne peut trouver de cause suffisante dans l'histoire passée, comme l'origine de la géométrie, de la tragédie ou de la démocratie. Les événements singuliers de la fondation, qu'ils soient de nature politique, religieuse ou artistique, ne se laissent pas enregistrer qu'en tant que tels. Comme le note Platon dans le dialogue Parménide ${ }^{25}$ (141 c-d), tout ce qui est dans le temps est plus jeune et plus vieux que luimême. Cela s'applique également aux immenses périodes temporelles du cosmos qui nous permettent de voir les étoiles, qui n'existent peut-être plus lorsque nous les découvrons dans le télescope. Seule une expérience dans laquelle tout reste fondamentalement le même serait épargnée par de tels décalages temporels.

Le retard (Nachträglichkeit) de nos réponses n'est rien d'autre qu'un simple manque. C'est seulement puisque cela a déjà commencé par nous lorsque nous commençons nousmêmes qu'un avenir s'ouvre à nous, qui signifie plus qu'une extension de notre propre présent et une extrapolation des orientations. Dans le mythe de la création humaine que Platon raconte dans Protagoras (320 c-322 e), les frères Prométhée et Épiméthée apparaissent. Prométhée, le célèbre héros de l'invention de la technique est, comme son nom l'indique, « celui qui réfléchit avant » (vorbedacht), contrairement à Épiméthée, 
qui est « celui qui réfléchit après » (nachbedacht) ; l'un prend des précautions, l'autre subit les conséquences. Mais l'homme répondant est en quelque sorte Prométhée et Épiméthée en une seule personne. Le Voraussehen et le Nachsehen ${ }^{26}$ s'entrelacent comme $^{2}$ le propre dans l'étranger et l'étranger dans le propre. Si la personne dans la force de l'âge marche sur deux pieds, la bipédie n'est pas totalement synchronisée. Edipe « pieds enflés» ne résout pas seulement le mystère du sphinx, il l'incarne aussi corporellement.

\section{Épilogue : Noms}

Nos réflexions sur la responsivité de l'homme seraient incomplètes sans un regard sur l'anonymat de l'homme. Il ne s'agit pas, en premier lieu, de noms génériques qui dénotent des caractéristiques générales ou de noms propres qui servent à identifier des individus. Ces noms sont attribués sous forme de codes ou de mots de passe. Mais s'il n'y avait que cela, le donneur de nom serait le maitre des noms (Herr der Namen). Luimême aurait une fonction de la même façon que le sujet transcendantal, mais pas de nom. Du nom de l'homme, il resterait seulement le nom générique, avec ses connotations changeantes qui spécifient le statut de l'homme. Que l'on parle de

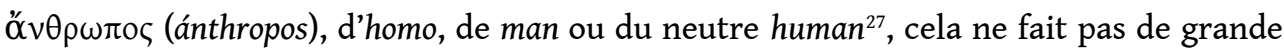
différence.

Mais l'homo respondens avec lequel nous avons affaire n'est pas seulement un porteur de nom, c'est un être singulier qui a reçu son nom des autres. Le nom est le signe d'un caractère irremplaçable. On célèbre les jours des noms, mais pas les jours des concepts. Avant que le nom soit disponible en tant que désignation nominale, il remonte à la surface comme nom usuel grâce auquel la personne adressée écoute et répond - ou ne répond pas. Un tel nom propre a les caractéristiques d'un nom étranger et un noyau d'anonymat. Il contient les traces d'une histoire nominale. C'est le cas d'ÆEdipe, qui a été abandonné par son père avec les pieds enflés, comme cela a déjà été mentionné. Cela est particulièrement évident dans les promesses des noms juifs, par exemple quand Abraham est considéré comme "Père d'une multitude (de peuples) » ou Isaac comme « il rira ». ${ }^{28} \mathrm{~L}$ ' " être vivant doté d'une raison » pourrait donc se transformer en un "être vivant qui répond à un nom ». Ce que cela signifie pour la singularité de l'homme devient clair dès que le nom est supprimé et remplacé par des chiffres et des numéros d'immatriculation comme pour les prisonniers des camps de concentration ou les esclaves à vendre. Cela nous amène dans le vaste domaine de la culture, de la politique et de l'éthique des noms. L'utilisation de noms dans les domaines non humains devrait également être envisagée, depuis la dénomination des animaux domestiques et des plantes rares jusqu'à la dénomination des ouragans et des étoiles rares. Le fait que l'homme réponde avec emphase ne signifie pas que tout le reste se contente de fonctionner ou d'obéir. La corporalité et l'appartenance au monde de l'homme comprend plutôt le fait que l'homme est sans cesse plus ou moins homme. Seules les personnes complètement normalisées seraient en paix avec elles-mêmes. 


\section{NOTES}

1. [Afin de rendre la présentation plus claire, nous avons mis entre crochets les remarques du traducteur. Les notes sans crochet sont de l'auteur].

[Le texte est initialement paru en allemand dans Sozialität und Alterität, Berlin, Suhrkamp, 2015, p. 15 à 28 (Copyright: (c) 2015, Berlin, Suhrkamp). Je remercie Clément Serniclay et Bernhard Waldenfels pour leurs remarques sur la traduction. N.D.T.].

2. Sur la diversité évolutive de l'homme, voir la description magistrale du paléontologue André Leroi-Gourhan, Le Geste et la Parole, T1. : Technique et langage, T2. : La mémoire et les Rythmes, Paris, Albin Michel, coll. «Sciences d'aujourd'hui », 1964-1965. Et sur les nouveaux développements de la recherche, M. Tomasello, Aux origines de la cognition humaine [1999], Paris, Retz, 2004, trad. par Y. Bonin.

3. Voir M. Bakhtine, "Das Wort im Roman ", in Die Ästhetik des Wortes, sous la direction de R. Grübel, Francfort-sur-le-Main, Suhrkamp, 1979, p. 185. Je mentionne en premier lieu le théoricien littéraire russe, car il est l'un des rares auteurs pour qui la "responsivité " («Antwortlichkeit») prétend à sa propre place face à la responsabilité (Verantwortlichkeit). Voir ensuite, par l'auteur, Vielstimmigkeit der Rede, Francfort-sur-le-Main, Suhrkamp,1999, p. 156-170.

4. [F. Nietzsche, Par-delà bien et mal [1886], § 62, in Friedrich Nietzsche: Euvres, Paris, Flammarion, 2000, p. 686, trad. par P. Wotling. N.D.T.].

5. [Waldenfels utilise la traduction d'Antigone en allemand réalisée par Hölderlin. Nous faisons donc le choix de laisser dans le cœur du texte cette version sur laquelle s'appuie l'auteur. Pour une meilleure compréhension, la version française du texte d'Hölderlin, traduite par LacoueLabarthe (L'«Antigone» de Sophocle, éd. bilingue franco-allemande, Paris, Bourgeois, 1978, p. 43-44) est la suivante : «Expert en tout, inexpert. Il n'arrive à rien. / Le lieu promis aux morts / seul, il ne sait le fuir.»

Waldenfels évoque les vers 360 à 362, il s'agit des vers 375 à 378 dans l'édition de LacoueLebarthe. N.D.T.].

6. [«Beaucoup est monstrueux. Rien cependant qui soit plus monstrueux que l'homme.» Antigone, V. 349-350, p. 43 de l'édition citée ci-avant. N.D.T.].

7. [Freud décrit l'auto-analyse, notamment l'interprétation des rêves, comme une descente «aux Enfers». En exergue de L'interprétation des rêves, il cite d'ailleurs une phrase de l'Enéide qui montre que le détour par l'inconscient peut être compris comme descente aux Enfers: «si je ne puis fléchir ceux d'en haut, je mettrai en branle l'Achéron. ». N.D.T.].

8. [I. Kant, Critique de la raison pure [1787], Paris, Baillières, 1869, trad. par J. Barni, p. 21-22. N.D.T.].

9. Voir Aristote, Politique, I, 2, § 5, où Aristote indique ici que si les outils travaillaient d'euxmêmes, les entrepreneurs n'auraient plus besoin d'ouvriers ni les maîtres d'esclaves. (Référence corrigée par le traducteur en accord avec l'auteur.)

10. Voir de l'auteur, Verfremdung der Moderne, Göttingen, Wallstein, 2001.

11. Voir J. Weizenbaum, Puissance de l'Ordinateur et Raison de l'Homme - du Jugement au Calcul [1976], Boulogne-sur-Seine, Éditions d'Informatique, 1981, trad. par M.-T. Margulici, Chapitre 7.

12. Voir de l'auteur, Grenzen der Normalisierung, Francfort-sur-le-Main, Suhrkamp, 1998.

13. Voir de l'auteur, Grundmotive einer Phänomenologie des Fremden, Francfort-sur-le-Main., Suhrkamp, 2006 et spécialement Findigkeit des Körpers, Dortmunder Schriften zur Kunst (Kataloge und Essays, V.1), 2004; ou bien Phänomenologie der Aufmerksamkeit, Francfort-sur-le-Main, Suhrkamp, 2004, Chapitre 6.

14. Voir K. Goldstein, Der Aufbau des Organismus, Den Haag, 1934. Nouvelle édition chez W. Fink, Paderborn, 2014. 
15. [I. Kant, Prolégomènes à toute métaphysique future qui pourra se présenter comme science [1783], Paris, Vrin, 1965, trad. par L. Guillermit, p. 170, note 1. On peut traduire cela par « la profondeur fertile de l'expérience ». N.D.T.].

16. Je me réfère à ma critique de la « raison juridique » : Schattenrisse der Moral, Francfort-sur-leMain, Suhrkamp, 2006.

17. [Sur les trois dernières possibilités, Waldenfels joue avec les préfixes du verbe fallen: « zufällt, auffällt oder einfällt ». N.D.T.].

18. [En français dans le texte. N.D.T.].

19. [F. Nietzsche, Ainsi parlait Zarathoustra [1892], "L'heure du suprême silence ", in Friedrich Nietzsche: Euvres, Paris, Flammarion, 2000, p. 450, trad. par G. Bianquis, revue par P. Mathias. N.D.T.].

20. [F. Nietzsche, Par-delà bien et mal, op. cit., p. 640. N.D.T.].

21. [Waldenfels utilise ces verbes de manière récurrente. Widerfahren signifie littéralement arriver contre. Dans le contexte de l'œuvre de Waldenfels, on le traduit généralement par « ce qui nous arrive ou nous heurte ». Nous avons choisi, par un souci de cohérence, de traduire auffallen par attirer l'attention durant tout le texte (cela peut aussi signifier frapper ou se remarquer). De même, nous rendons einfallen par venir à l'esprit (cela peut aussi signifier envahir ou rentrer). N.D.T.].

22. [Goethe, Torquato Tasso [1790], in Euvres complètes T. 3, Paris, Hachette, 1870, trad. par J. Porchat, p. 375. N.D.T.].

23. [Pathei Mathos : cela désigne un enseignement par l'épreuve. N.D.T.].

24. [Décalage temporel. N.D.T.].

25. [Notamment : «Parménide - Ainsi donc ce qui devient plus vieux que soi devient nécessairement en même temps plus jeune que soi. ", Platon, Parménide, Bibliothèque numérique artyuiop, trad. par : E. Chambry, p. 48. N.D.T.].

26. [Littéralement: voir avant et voir après. Dans la langue courante, on traduit généralement voraussehen par prévoir, et nachsehen par vérifier. N.D.T.].

27. ["man" et "human" sont en anglais dans le texte. N.D.T.].

28. Voir S. Mosès, L'Éros et la Loi, lectures bibliques, Seuil, 1999, particulièrement le chapitre 2 sur le rôle du nom dans le dialogue. Voir aussi, de l'auteur, Das Zwischenreich des Dialogs, Den Haag, Nijhoff, 1971, p. 284-288. 